\title{
La circulation des normes dans le domaine du blanchiment d'argent : le rôle du G7/8 dans la création d'un régime global
}

\section{Amandine Scherrer}

\section{(2) OpenEdition \\ Journals}

Édition électronique

URL : http://journals.openedition.org/conflits/2069

DOI : $10.4000 /$ conflits.2069

ISSN : 1777-5345

Éditeur :

CCLS - Centre d'études sur les conflits lilberté et sécurité, L'Harmattan

Édition imprimée

Date de publication : 1 juin 2006

Pagination : 130-148

ISBN : 2-296-01073-3

ISSN : 1157-996X

Référence électronique

Amandine Scherrer, "La circulation des normes dans le domaine du blanchiment d'argent : le rôle du G7/8 dans la création d'un régime global », Cultures \& Conflits [En ligne], 62 | printemps 2006, mis en ligne le 19 juillet 2006, consulté le 30 mars 2021. URL : http://journals.openedition.org/conflits/2069 ; DOI : https://doi.org/10.4000/conflits.2069

Ce document a été généré automatiquement le 30 mars 2021

Creative Commons License 


\title{
La circulation des normes dans le domaine du blanchiment d'argent : le rôle du G7/8 dans la création d'un régime global
}

\author{
Amandine Scherrer
}

1 Si les définitions du blanchiment d'argent varient, un élément commun les caractérise ${ }^{1}$ : le transfert de biens acquis illégalement dans le système économique légal ${ }^{2}$. La notion d'argent "sale", qui induit des pratiques de blanchiment et de recyclage, est commune à la plupart des définitions nationales et internationales : on parle de blanchiment en français, de money laundering en anglais, de Gewuldwäsche en allemand, de blanqueo en espagnol...

Depuis la fin des années 1990, il y a eu une mobilisation croissante au niveau international contre cet "argent sale», conduisant à la mise en place de ce que beaucoup qualifient de régime global, c'est-à-dire un "ensemble de principes, de normes, de règles et de procédures de décision, implicites ou explicites, autour desquels les attentes des acteurs convergent dans un domaine spécifique ${ }^{3}$ ». La majeure partie de la littérature liée au thème du blanchiment d'argent ${ }^{4}$ s'est largement intéressée aux instances internationales qui ont pris en charge cette « lutte » et à leurs outils de mise en oeuvre: les directives européennes, les recommandations du GAFI (Groupe d'action financière internationale), les conventions onusiennes, etc. Les analyses ont été d'ordre juridique, économique, ou encore sociologique, et ont parfois pointé les défaillances, les incohérences ou encore les insuffisances de ce régime global. Ces publications partent presque toutes d'un consensus présupposé sur la démarche à suivre au niveau international afin de remédier à ce "mal» que constituerait le blanchiment d'argent, perçu comme le nerf des organisations criminelles en tous genres, et, plus récemment, des groupes terroristes. En revanche, la construction de ce consensus et les acteurs qui en sont à l'origine sont plus ou moins occultés dans ces mêmes publications, au profit de la diffusion de cette démarche proactive vis-à-vis d'un 
argent impie. Cet aspect est pourtant essentiel pour la compréhension de l'élaboration de ce régime global, qui, malgré ce que cette appellation peut suggérer (une convergence), résulte d'un consensus somme toute assez «mou», qui cache une concertation parfois houleuse d'acteurs d'origines diverses. Dès lors, un travail de reconstitution sociologique des acteurs mobilisés dans la lutte anti-blanchiment permet de décortiquer ces normes internationales ${ }^{5}$ qui ont été préférées à un moment donné, de comprendre les jeux de ces acteurs selon les sphères dans lesquelles ils interagissent, et les enjeux qui en découlent.

3 Nous tenterons, dans cet article, de compléter les lacunes de la littérature existante, en opérant un repérage des acteurs, et en les replaçant dans le contexte particulier qui est le leur (au regard de leurs formations, de leurs ressources, de leurs statuts), afin de comprendre les normes internationales ayant trait à la lutte contre le blanchiment d'argent. En ce sens, nous avons fait le choix de nous appuyer plus précisément sur l'exemple du G7/8 ${ }^{6}$, créé en 1975 et dont le rôle n'a cessé d'évoluer depuis. Trop souvent méconnue et peu étudiée, cette instance a eu pourtant une influence considérable sur la constitution d'un régime global anti-blanchiment. Il s'agira notamment de montrer que l'Union européenne n'est pas isolée dans ce domaine, que les normes élaborées par cette instance ne lui sont pas toujours propres et qu'il existe des influences multiples provenant d'autres enceintes. Il ne s'agit pas ici de remettre en cause le rôle fondamental qu'ont eu les initiatives européennes, notamment au sein du Conseil de l'Europe dès la fin des années 1980, et leur influence sur ce régime global spécifique, mais plutôt de montrer l'effet de circulation des normes entre différentes instances internationales. La démarche proposée tendra en conséquence vers deux objectifs. Une analyse classique visera, dans un premier temps, à mettre en lumière ce qui se fait au sein du G7/8 en matière de lutte anti-blanchiment et quels en sont les principaux acteurs. Une analyse sociologique de ces mêmes acteurs aidera ensuite à relever leur multipositionnement au niveau international, mais aussi les lignes de convergence, ou de clivage, qui les unissent et/ou les séparent. Cette mise en perspective approfondira la compréhension des discours et des normes institués au sein du régime global anti-blanchiment.

Le G7/8 et son insertion dans la lutte internationale contre le blanchiment d'argent

4 On ne peut analyser correctement le rôle du G7/8 dans la lutte anti-blanchiment sans replacer celui-ci dans son contexte socio-historique et, en premier lieu, en prenant acte des déclarations et des communiqués livrés par les chefs d'Etat et de gouvernement à l'issue de chacune de leurs rencontres.

5 La question du blanchiment d'argent a été abordée pour la première fois dans le cadre du G7/8 en 1989, lors du sommet de Paris, à un moment où il s'agissait de lutter contre le trafic de stupéfiants. Sous une section spécifique appelée «Problèmes relatifs aux stupéfiants ", la déclaration finale du sommet ${ }^{7}$ implique les pays du G7 dans un effort commun visant à « s'opposer à la production de stupéfiants, [...] réduire la demande et poursuivre la lutte contre le trafic lui-même et le blanchiment des gains qu'il procure ». Le but poursuivi étant de "faciliter», aux niveaux national et international, « l'identification, la détection, le gel, la saisie et la confiscation des gains illégaux de la drogue ». C'est lors de ce même sommet que les pays du G7 convoquent :

6 « un groupe spécial d'experts financiers comprenant les participants au sommet et d'autres pays intéressés par ces problèmes. Son mandat est d'évaluer les résultats de la coopération mise en ouvre afin de prévenir l'utilisation du système bancaire et des 
institutions financières aux fins de blanchir l'argent et d'étudier des mesures préventives supplémentaires dans ce domaine, y compris l'adaptation des systèmes juridiques et réglementaires de façon à renforcer l'entraide judiciaire multilatérale ».

7 C'est la naissance du GAFI, qui délivre son premier rapport au sommet de Houston l'année suivante. Au sommet de Londres en 1991, les participants au G7 approuvent l'action du GAFI et acceptent que ce dernier fonctionne sur une base permanente, avec un secrétariat assuré par l'OCDE. Ainsi, dans un premier temps, les pays membres du G7 abordent la question du blanchiment d'argent sous l'angle spécifique du seul trafic de stupéfiants.

8 C'est en 1994, au sommet de Naples, que la notion de « crime transnational » apparaît dans les communiqués officiels du G7 ${ }^{8}$. La lutte contre le blanchiment d'argent prend alors indéniablement une nouvelle dimension : de la lutte contre les « gains illégaux de la drogue », on parvient à la volonté d'empêcher « le blanchiment des produits du trafic de stupéfiants et autres délits graves ou autres infractions portant sur des sommes importantes ». Lors du sommet d'Halifax en 1995, les Sept décident d'ailleurs d'élargir leur action en faisant de la criminalité transnationale organisée un thème de travail à part entière. En ce sens, ils proposent, à l'initiative canadienne, la mise en place d'un groupe d'experts sur la criminalité transnationale organisée, dont les travaux seront remis un an plus tard, lors du sommet de Lyon de 1996. Le "crime transnational », sans qu'aucune définition précise ne soit pour autant donnée, est reconnu comme un «problème mondial ». Au sommet de Denver en 1997, les pays du G7 se saisissent de questions désignées comme "globales"; c'est d'ailleurs le titre qui est donné à une section spéciale de la déclaration finale ${ }^{9}$, qui traite de thèmes aussi divers que l'environnement, la sûreté nucléaire, les questions énergétiques, le clonage humain, la criminalité transnationale organisée, les drogues illicites ou encore le terrorisme. Dans cette liste, la lutte contre la criminalité transnationale organisée est affichée et reconnue comme " une priorité ». Le communiqué du sommet de Birmingham en 1998 ${ }^{10}$ est encore plus explicite : les participants du G7/8 se sont penchés « sur trois grands impératifs auxquels le monde est confronté en cette veille de XXIe siècle ", que sont " une croissance et un développement économiques durables, la création d'emplois et la lutte contre l'exclusion sociale, combattre le trafic des drogues et la criminalité transnationale, qui menacent de saper cette croissance, portent atteinte à la primauté du droit et minent des vies humaines dans tous les pays du monde ».

9 Les termes employés lors de ce sommet sont particulièrement forts: «[...] la mondialisation a entraîné dans son sillage une hausse spectaculaire de la criminalité internationale. Celle-ci revêt des formes multiples, dont la contrebande des armes et des drogues, le trafic des êtres humains, l'utilisation des nouvelles technologies pour voler, frauder et contourner la loi, ainsi que le blanchiment des profits du crime. Non seulement ces crimes posent-ils une menace à nos propres citoyens et à leurs collectivités parce que des vies sont minées par la drogue et que les sociétés vivent dans la crainte du crime organisé, mais ils constituent aussi une menace mondiale qui risque de saper le fondement démocratique et économique des sociétés du fait de l'investissement de capitaux illégaux par des cartels internationaux, de la corruption, de l'affaiblissement des institutions et de la perte de confiance dans la primauté du droit . [...] Il ne doit y avoir de refuge sûr ni pour les criminels ni pour leur argent ».

Le blanchiment d'argent, après avoir été traité exclusivement sous l'angle de la lutte contre le trafic de stupéfiants, s'élargit donc à d'autres délits, assimilés sous le vocable 
général de CTO (l'acronyme pour "Criminalité transnationale organisée » est utilisé tel quel dans les communiqués officiels à partir de 2000). Les attentats du 11 septembre 2001 amènent un troisième glissement : l'inclusion du terrorisme dans la notion de СTO et le recentrage de la lutte contre le blanchiment sur les actions terroristes. Le 19 septembre 2001, les participants au G7/8 présentent une déclaration conjointe qui condamne les attentats ${ }^{11}$. La priorité clé reste la mise en ouvre globale de la résolution 1373 du Conseil de Sécurité des Nations unies et des douze conventions onusiennes contre le terrorisme ${ }^{12}$. A travers les actions recommandées par le $G 7 / 8$, on note la volonté de s'attaquer au financement du terrorisme. En ce sens, le G7/8 encourage le GAFI à réviser ses recommandations. Ce dernier point marque un pas supplémentaire dans l'analyse: le lien organique existant entre le GAFI, figure de proue dans l'élaboration de normes internationales anti-blanchiment, et le G7/8.

Le GAFI : le " protégé » des acteurs du système G7/8 en matière de lutte antiblanchiment

11 Bien qu'utile par ailleurs, l'une des grandes failles de la littérature académique sur le G7/8 réside dans la considération de cette instance comme un tout homogène, un acteur unitaire. Si les sommets du G7/8 ne sont plus étrangers aux médias et à l'opinion publique, les rencontres annuelles des chefs d'Etat et de gouvernement - ce que beaucoup perçoivent comme un club fermé et exclusif - dissimulent en revanche tout un processus de préparation en amont, qui fait intervenir un grand nombre d'acteurs demeurant, pour ainsi dire, dans l'ombre des communiqués officiels et médiatisés. Le G7/8 est davantage un système, dont les rouages se situent au coeur des administrations nationales des pays membres. L'inflation de l'agenda du G7/8 depuis sa création en $1975 \mathrm{a}$, en effet, conduit progressivement à une division du travail, qui se coordonne au niveau ministériel et au niveau des groupes d'experts sur chaque sujet traité, avant de « remonter » aux sommets par l'intermédiaire des « sherpas » ${ }^{13}$, autant de sommets qui ne constituent finalement que la partie visible d'un mécanisme désormais bien huilé ${ }^{14}$. Mais cette méconnaissance des mécanismes opératoires au sein des instances coordinatrices et initiatrices du G7/8 conduit malheureusement à occulter ou sous-estimer l'activité du G7/8 en matière de lutte contre le blanchiment d'argent.

Cette mobilisation a été entreprise à des niveaux variés de la machine G7/8, contribuant ainsi pleinement à la mise en oeuvre de normes à vocation internationale élaborées au sein du G7/8, sans que le rôle de ce groupe ne soit forcément visible. Si les déclarations et les communiqués de fin de sommets peuvent laisser à penser que le G7/8 a délégué son initiative anti-blanchiment au GAFI, en se contentant de temps à autre d'impulser des mesures complémentaires, elles atténuent considérablement le rôle des acteurs internes à la machine G7/8, qui, eux, continuent d'avoir un rôle d'influence sur les travaux menés au sein du GAFI. En effet, si en 1989 lors du sommet de Paris, l'initiative de la création du GAFI et la mise à l'agenda de la question du blanchiment semblent avoir émergé "spontanément", essentiellement à l'initiative américaine dans le contexte de "guerre contre la drogue ", il y a eu par la suite une délégation croissante de cette initiative au niveau ministériel, qui s'est de plus en plus autonomisée au cours des années 1990, parallèlement, mais en connivence constante avec le GAFI. Aussi, c'est sans conteste les ministres des Finances et les gouverneurs des banques centrales du G7 ${ }^{15}$, qui ont été les plus prolifiques dans la mise en place des réseaux internationaux de lutte anti-blanchiment, allant de la création de cellules de renseignement financier en 1998 (reliées entre elles au sein du groupe Egmont) et de 
l'adoption de mécanismes de déclaration de soupçons (TRACFIN), à la création du Forum de stabilité financière en 1999 et à l'inclusion des Institutions financières internationales - IFI - (FMI et Banque mondiale) dans la lutte anti-blanchiment. Par ailleurs, ce sont ces mêmes ministres des Finances qui présentent des recommandations dans des rapports, comme ceux dits de "Fukuoka» (en vue de la préparation du sommet d'Okinawa en 2000) et de « Rome » (en vue du sommet de Gênes en 2001), destinées à d'autres forums de discussion et d'action au niveau international. Le GAFI est ainsi largement utilisé par les ministres des Finances et les gouverneurs des banques centrales du G7 pour diffuser leur message et promouvoir une mobilisation internationale. Les déclarations de ces réunions appuient, en effet, non seulement l'action du GAFI, mais orientent aussi ses travaux. C'est ainsi que le plan d'action pour combattre le financement du terrorisme, adopté par les ministres au lendemain des attentats du 11 septembre 2001 16, "encourage » le GAFI à adopter de nouvelles recommandations sur le financement du terrorisme, donnant lieu aux neuf recommandations spéciales ${ }^{17}$.

Les ministres de la Justice et de l'Intérieur du G8 sont également actifs dans la promotion et l'orientation de la lutte anti-blanchiment sur le plan international. La participation proactive des "gatekeepers" (avocats, comptables, etc.) a par exemple été d'abord envisagée lors d'une réunion ministérielle des ministres de l'Intérieur et de la Justice du G8 en $1999{ }^{18}$. Cet élément fut repris un an plus tard par les ministres des Finances du G7 ${ }^{19}$, qui «demandent » au GAFI d'envisager la possibilité de revoir ses quarante recommandations pour tenir compte de ces enjeux, enjeux inclus une année plus tard dans les neuf recommandations spéciales. Bien que le GAFI ait acquis une certaine autonomie depuis sa création, le G7/8 conserve une influence incontestable sur le groupe, via ses échanges au niveau ministériel. Un premier groupe d'acteurs d'importance $\mathrm{du} G 7 / 8$ se dégage donc de ces remarques préliminaires : les ministres des Finances du G7 d'une part, ceux de la Justice et de l'Intérieur du G8 d'autre part. Leur rôle dans la diffusion de normes anti-blanchiment, notamment et surtout via le GAFI, ne doit pas être minimisé. Un deuxième groupe d'acteurs est également identifiable au sein de la structure G8: les groupes de travail, nommés officiellement groupes d'» experts » du G8. Deux de ces groupes ont ici une importance significative : le groupe de Lyon et le groupe de Rome.

Le Groupe d'experts sur la criminalité transnationale organisée du G8 a été créé à l'initiative des chefs d'Etat et de gouvernement lors du sommet d'Halifax en 1995. Il prend le nom de "groupe de Lyon" en 1996, puisqu'il soumet cette année-là au sommet de Lyon ses quarante recommandations, sur le même modèle que celles du GAFI. Ses participants sont des fonctionnaires issus des ministères de l'Intérieur, des Affaires étrangères, de la Justice et des douanes des différents pays du G8. Dans les premières recommandations du groupe de Lyon de 1996, ainsi que dans leur version révisée de 2002, les experts G8 entendent ne pas empiéter sur les activités du GAFI, mais se proposent d'apporter une expertise supplémentaire dans certains domaines, notamment sur les aspects judiciaires et policiers ${ }^{20}$. Les travaux du groupe de Lyon sont relayés au niveau des rencontres des ministres de la Justice et de l'Intérieur du G8. Depuis 2001, les réunions des experts du groupe de Lyon se font de manière quasi conjointe avec celles des experts du groupe de Rome, le groupe d'experts G8 sur le terrorisme, créé en $1982{ }^{21}$. Les recommandations du G8 sur le contre-terrorisme, adoptées en $2002{ }^{22}$, ont été élaborées par les experts du groupe de Rome dans le but explicite « de fournir un complément aux quarante recommandations du groupe du G8 
sur la criminalité transnationale organisée ». Une section spécifique y est consacrée au financement du terrorisme, qui vise plus particulièrement les mesures de confiscation et de gel des avoirs terroristes ainsi que la traçabilité des fonds détenus par des individus et/ou des groupes qualifiés de terroristes.

Les groupes ministériels (des Finances, de la Justice et de l'Intérieur) et les groupes d'experts du G8 constituent un ensemble d'acteurs qui travaillent parallèlement au GAFI, mais également en collaboration étroite. C'est en ce sens que l'on peut considérer le GAFI comme «le protégé » du G7/8. Le GAFI est ainsi largement utilisé par le G7/8 pour la promotion de ses propres normes, et la complicité G7/8-GAFI a été cruciale dans la constitution de ce régime global anti-blanchiment. Le nombre des membres du GAFI n'ayant cessé d'augmenter depuis la création de celui-ci, on peut dire que le G7/8 peut ainsi largement diffuser son message ${ }^{23}$. La teneur et l'internalisation de ce message peuvent s'assimiler à une socialisation des normes G8, entendue comme "l'inclusion de nouveaux membres [...] dans un type de comportements qui est préféré dans une société ${ }^{24}$ ", au sein du GAFI et plus largement au sein de la communauté internationale. Le G7/8 : un diffuseur de normes sur la scène internationale La spécificité du G7/8 (absence de statut juridique, de secrétariat et de mécanisme de coercition) mène à le considérer comme une institution au sens social, c'est-à-dire comme un groupe qui crée un rapport social ayant un certain nombre d'effets et de contraintes. Un des effets les plus importants dans le cadre de cet article est le processus de concertation, qui permet de parvenir à l'élaboration de règles et de normes communément admissibles et admises. Dès lors, la contrainte réside dans le respect de ces normes et, concrètement, dans l'internalisation de ces dernières dans les espaces nationaux des pays membres. Ces normes opèrent par conséquent un mouvement circulaire à deux niveaux : elles atteignent à la fois les pays membres du G8 et elles sont également destinées à avoir une portée universelle, au-delà des Etats membres, notamment dans d'autres instances internationales. Dans le domaine antiblanchiment, le cas de la Russie constitue pour le premier mouvement un exemple frappant d'une socialisation des normes élaborées en G7/8.

Pendant longtemps, en effet, la différenciation G7/P8 a prévalu, le «P » de «P8 » étant utilisé pour «Political». Le G7, à l'origine, regroupait les sept pays les plus industrialisés ${ }^{25}$ et quand il fut question d'y intégrer la Russie au début des années 1990, les Sept optèrent pour l'octroi d'une participation accrue graduellement à chaque sommet successif. Cependant, il était globalement acquis pour les membres initiaux que la Russie serait exclue des débats économiques. Cette participation graduelle conduisit alors à la formation d'un P8, qui traitait non seulement des questions de sécurité et de politique conventionnelles, mais aussi de toute une gamme de problèmes globaux ou de "sécurité nouvelle», comme le crime transnational, les drogues, le terrorisme et la protection environnementale. Cependant, depuis 1998, lors du sommet de Birmingham, la distinction G7/P8 a été abandonnée au profit de la dénomination officielle de « G8 ». La Russie accueillera d'ailleurs pour la première fois le sommet du G8 en juillet 2006, à Saint-Pétersbourg. Un élément est ici à remarquer : alors que les ministres des Finances du G7 continuent aujourd'hui de se rencontrer sans la Russie concernant les questions macro-économiques, la Russie y est en revanche complètement intégrée lors des discussions concernant la lutte anti-blanchiment. Elle a, en outre, organisé la rencontre des ministres de la Justice et de l'Intérieur du G8 à Moscou en 1999. Elle est, enfin, partie prenante du second groupe d'acteurs du système G8 identifié précédemment : les 
groupes d'experts de Lyon et de Rome. La lente intégration de la Russie au processus du G8 a conduit celle-ci à s'impliquer davantage dans la lutte contre le crime organisé et, par conséquent, dans la lutte contre l'argent sale. La Russie, à cet égard, a opéré une internalisation expresse des normes du GAFI : incluse dans la liste noire des " pays et territoires non coopératifs ${ }^{26}$ » du GAFI en 2000, elle est retirée de la liste en 2002 et devient membre à part entière du GAFI en 2003. Elle se trouve donc désormais intégrée au régime global anti-blanchiment ${ }^{27}$.

18 Dans le deuxième mouvement circulaire, les orientations définies en G7/8 ont marqué de leur sceau les recommandations du GAFI. Or, le GAFI constitue le pilier central du régime global actuel anti-blanchiment. Ce groupe est la première instance à avoir, dès le début des années 1990, adopté des recommandations à portée universelle. Ces recommandations se regroupaient, à l'origine, sous trois objectifs centraux :

- l'amélioration des systèmes légaux nationaux anti-blanchiment, plus spécifiquement en droit pénal ;

- le renforcement de la coopération internationale ;

- l'extension du rôle du système financier dans la lutte anti-blanchiment ${ }^{28}$.

22 Ces quarante recommandations et leur version révisée de 1996 ont largement influencé les initiatives qui ont suivi, au niveau international mais aussi au niveau régional, notamment au sein de l'UE. C'est ainsi que ces recommandations de soft law ont été depuis largement diffusées dans des régimes légaux contraignants, comme c'est le cas des directives européennes. Si on peut parler de régime global au sens où Krasner l'entendait, c'est parce que se sont mises progressivement en place des normes à portée universelle reconnues comme des standards de référence. A première vue, on lit d'ailleurs une grande cohérence dans ce régime, tant au niveau des perceptions de la menace que représente l'argent sale qu'au niveau des outils reconnus comme nécessaires dans la lutte contre le blanchiment des biens illégaux.

Le rôle du G7/8 dans la construction de cette cohérence n'est pas anodin. La circulation de ses normes est, en effet, amplement facilitée par le fait que cette enceinte constitue une plateforme communicationnelle de choix. Faisant intervenir des participants de quatre pays de l'UE (qui interviennent également lors des réunions des ministres du Conseil de l'Europe), d'un pays asiatique influent - le Japon ${ }^{29}-$, de l'Amérique du Nord et de la Russie, les rencontres en G7/8 ont une importance significative pour la diffusion de messages. On oublie également trop souvent l'implication d'autres instances au processus $\mathrm{G} 7 / 8$, notamment l'UE, qui est de facto le neuvième membre du $\mathrm{G} 7 / 8^{30}$. Toutes les réunions préparatoires aux sommets $\mathrm{du} \mathrm{G} 7 / 8$ font ainsi participer et intervenir des membres de la Commission européenne, et la délégation de l'UE est très présente dans les débats. Une délégation JAI ${ }^{31}$ de l'UE est ainsi systématiquement représentée lors des rencontres ministérielles de la Justice et de l'Intérieur du G8, jouant un rôle d'observateur. A cet égard, certains membres de ces délégations européennes reconnaissent le rôle central du G7/8, notamment dans sa capacité à impulser ce qui se fait au sein de l'UE et à inclure dans la coordination internationale des pays comme la Russie, le Japon, le Canada et, plus important encore, les Etats-Unis. Si nous avons montré jusqu'ici que le système $\mathrm{G} 7 / 8$, à travers ses groupes ministériels et ses groupes d'experts, exerçait sur le GAFI un degré d'impulsion non négligeable, qui s'étendait également, par effet de ricochet, sur la scène internationale, un deuxième niveau d'analyse s'avère nécessaire pour mettre en lumière les rouages de la 
constitution de ce régime global. Si l'on peut parler de cohérence, de consensus, comment alors se sont-ils constitués ? Comment, d'ailleurs, mesurer leur solidité ? La lutte contre l'argent sale est abordée le plus souvent à travers des approches institutionnelles, et on parle le plus souvent de l'action du GAFI, du G7/8, de l'ONU ou de l'UE, en les appréhendant comme des entités abstraites, sans tenir compte de leurs structures internes et des liens qui s'opèrent entre elles. Il y a pourtant une vraie valeur heuristique à comprendre qui sont les acteurs à la source de cette production normative, à passer dans l'envers du décor afin de saisir comment s'est opérée la circulation de ces normes. C'est pourquoi il convient de se pencher davantage sur les acteurs du système G7/8 et, par extension, sur ceux du GAFI et de l'UE en s'attardant davantage sur ces « experts », fonctionnaires des administrations des pays du G7/8, qui interviennent dans les délégations ministérielles et/ou dans les groupes de travail. Retrouver ici les qualités et les trajectoires des acteurs impliqués permet d'ébaucher les premiers éléments de réponse. Cette approche de type sociologique vise à mettre en perspective nos premiers commentaires. Il s'agit de savoir qui sont les acteurs mobilisés dans la lutte anti-blanchiment, de les identifier et de les catégoriser à l'aune de leur formation, de leur positionnement, de leurs ressources. Ce questionnement permettra de comprendre l'effet d'imposition et de circulation des normes antiblanchiment.

Les acteurs du G7/8 dans la lutte anti-blanchiment : effet de mobilisation et de mobilité Dans cette tentative d'identification des acteurs du système G8 mobilisés dans la lutte anti-blanchiment, ont été mis en exergue les groupes ministériels et les groupes d'experts, les chefs d'Etat et de gouvernement ne faisant que légitimer davantage et officiellement tout ce travail préparatif.

Un premier effort de découpage permet de distinguer ces experts des acteurs politiques. Si les réunions ministérielles et les sommets du G8 font parler les ministres et les chefs d'Etat et de gouvernement, permettant ainsi une voix et la valorisation d'une approche commune au G7/8, ce sont les experts de ces délégations et de ces groupes de travail qui façonnent et forgent ce discours politique. Ces experts sont des fonctionnaires issus des administrations des pays du G7/8 qui préparent et coordonnent les rencontres au niveau gouvernemental. Ils peuvent être définis comme "des professionnels disposant d'une compétence reconnue dans un domaine particulier et qui revendiquent avec autorité leur connaissance politique dans ce domaine ${ }^{32}$ ». " Mandatés » par les pays membres du G7/8, ces acteurs assurent la coordination internationale en fournissant les informations et les conseils dont les acteurs politiques ont besoin ${ }^{33}$. Leur compétence leur est reconnue avant tout par les expériences qu'ils ont acquises au cours de leur carrière dans les domaines du crime organisé et du terrorisme (et, par extension, du blanchiment d'argent). Cependant, si certains de ces acteurs ont une vraie compétence, forgée par une longue expérience au sein de différentes enceintes, d'autres reconnaissent participer à ces groupes de travail internationaux sans connaissances précises ou préalables de ces problématiques. Certains types de postes constituent une porte d'entrée systématique à ces délégations, à ces groupes de travail, mais ils ne sont parfois que d'une courte durée. Se pose alors la question de la formation de certains de ces experts, qui acquièrent ce statut par le poste qu'ils occupent, et non par leur compétence en ce domaine. Certains admettent qu'ils se forment "sur le tas ", et qu'ils s'en remettent à la connaissance des " anciens " pour les aspects très techniques de la lutte contre le blanchiment des capitaux et contre le financement du terrorisme. C'est notamment le cas des diplomates impliqués dans 
ces négociations internationales, qui disposent certes d'un savoir assez général et peu technique ${ }^{34}$, mais qui savent imposer leur présence dans le champ de l'expertise internationale ${ }^{35}$.

Néanmoins, de manière générale, la lutte contre le crime organisé et le terrorisme - le volet anti-blanchiment constituant un de ses traits les plus importants - a vu l'émergence ainsi que la reconnaissance de ces experts. Et sans conteste, ces experts nationaux acquièrent dans une large mesure ce statut par leur participation aux négociations internationales. Ces «techniciens du crime » peuvent se regrouper sous trois catégories : les experts financiers, les experts judiciaires et les experts policiers. $\mathrm{Au}$ sein de la structure G7/8, ils sont aisément identifiables: les experts financiers oeuvrent dans les groupes de travail sur la criminalité financière, les experts judiciaires dans le sous-groupe de coopération judiciaire des groupes de Lyon et de Rome, les policiers dans le sous-groupe Law Enforcement de ces mêmes groupes. Presque tous sont également actifs au sein des groupes de travail de l'UE, et/ou de la Banque mondiale, du FMI, de l'ONU, de l'OCDE. Les experts financiers se retrouvent essentiellement dans les groupes de travail du G7/8 des finances traitant de la criminalité financière et au sein du GAFI. Par exemple, le secrétaire exécutif du GAFI en 2005, fonctionnaire au sein du ministère des Finances en France, avait effectué auparavant un passage à la Banque mondiale, en tant que spécialiste du secteur financier du département de l'intégrité des marchés financiers, après avoir été adjoint au chef de la délégation française au groupe de travail du G7 sur la criminalité financière, à la délégation française au GAFI et au groupe de travail de l'OCDE sur la corruption des officiels publics. Il avait été également chargé de la rédaction des projets de loi anti-blanchiment français, finalement adoptés en mai 2001. Le chef de la délégation française au GAFI en 1999, actuellement chef du service du financement de l'économie à la Direction générale du Trésor et de la politique économique, a travaillé étroitement à l'ECOSOC sur le thème de la corruption, a été membre du groupe de travail de l'OCDE sur la corruption, ainsi que membre du groupe de travail sur les centres off-shore au sein du Forum de stabilité financière, mis en place par les ministres des Finances du G7. C'est ce type de profils qu'on trouve parmi les experts financiers institutionnels. Ce sont ces acteurs qu'on retrouve à des niveaux différents de leur carrière, ou simultanément, au sein d'enceintes diverses comme le G7, l'ECOSOC, l'OCDE, le GAFI, l'UE, la BM, le FMI.

Les experts judiciaires participent, quant à eux, aux sous-groupes de coopération judiciaire des groupes de Lyon et de Rome. Ils gravitent également d'une instance internationale à l'autre. Le responsable du réseau judiciaire européen en France en 2003 coordonnait, par exemple, dans le même temps le groupe coopération judiciaire et le groupe de Lyon. En 1995, le chef de la délégation italienne au groupe de Lyon pour les questions légales et institutionnelles a été, par la suite, le président du Comité ad hoc des Nations unies pour l'élaboration de la convention sur la criminalité transnationale organisée. Cinq magistrats de liaison européens étaient, en outre, présents dans les délégations des pays du G8 lors de la rencontre des ministres du G8 de la Justice au sommet d'Evian en 2003. La plupart des magistrats qui occupent des postes nationaux stratégiques (notamment au sein des bureaux spécialisés dans le crime organisé, le blanchiment d'argent et le terrorisme au sein de leur administration de tutelle comme il en existe dans certains Etats ${ }^{36}$ ) sont amenés à circuler dans des lieux divers de négociations internationales, que ce soit au sein de l'UE, du G8 ou encore de l'ONU. Les experts policiers participent essentiellement au sous-groupe Law Enforcement des 
groupes de Lyon et de Rome. Ils se retrouvent également au sein du CTAG (CounterTerrorism Action Group), créé par le G7/8 au sommet d'Evian en 2003, qui regroupe des membres du G8, de la Commission européenne et du Comité contre le terrorisme des Nations unies. De la même manière que pour les experts judiciaires, un pôle spécialisé sur les questions de crime organisé, de blanchiment d'argent et de terrorisme permet l'accès systématique aux enceintes internationales, et les officiers de police qui appartiennent à ce type de pôle sont amenés à circuler au sein de divers forums internationaux ${ }^{37}$. C'est ainsi que les policiers membres des administrations européennes participant aux groupes de travail du G8 se retrouvent lors des groupes de travail de l'UE. Ce va-et-vient institutionnel, du national à l'international, à travers diverses instances internationales, a permis la constitution d'une communauté internationale d'experts. La nature des problématiques traitées, leur routinisation au sein des agendas internationaux et l'impératif de coopération international, tel qu'il s'est constitué depuis les années 1980, ont permis à ces acteurs de se rencontrer fréquemment (les participants aux groupes de Lyon et de Rome se retrouvent ainsi trois fois par an et communiquent régulièrement), de multiplier les points de contact entre eux et de contribuer ainsi à la mise en place de réseaux efficients. Ces réseaux d'experts facilitent une interaction répétée, permettant ainsi un espace de concertation horizontal, qui sociabilise ses participants au-delà des frontières nationales. Parmi ces acteurs internationaux mobilisés contre le blanchiment d'argent, beaucoup reconnaissent la valeur ajoutée que constitue le G7/8 dans l'élaboration de normes internationales. Un expert du groupe de Lyon en France participant au sous-groupe de coopération judiciaire parle, par exemple, du G7/8 comme "d'une enceinte de coordination d'Etats influents " et souligne que ce qui se fait en $\mathrm{G} 7 / 8$ "se retrouve automatiquement sur le plan international ». Un autre expert britannique assimile le G7/8 à un « laboratoire d'idées", un «facilitateur de contacts». De nombreux experts $\mathrm{du}$ groupe de Lyon accordent une valeur particulière à ces rencontres en G8 et y trouvent de nombreux avantages : ces réunions de travail permettent de s'accorder sur des aspects très pragmatiques, de décanter des obstacles opérationnels, de nouer des contacts informels mais récurrents avec des acteurs variés, de traditions et de cultures différentes, et de s'échanger et de confronter des pratiques. Surtout, beaucoup reconnaissent que le G7/8 constitue une des seules enceintes qui permette la discussion d'enjeux multilatéraux entre partenaires américains, européens, russes, japonais et canadiens. Le G7/8 permettrait en ce sens aux Etats-Unis de faire passer des messages à ses partenaires. Certains acteurs de la Commission européenne présents à ces groupes de travail en G8 soulignent, quant à eux, le fort degré d'expertise de ces réunions, et, surtout, la flexibilité des discussions qui s'y tiennent, en les opposant à la lenteur des processus européens. Issus des Finances, de la Justice, de l'Intérieur, ces acteurs sont des habitués des réunions de travail internationales et se retrouvent d'un groupe à l'autre, au sein de différentes enceintes: des groupes de travail en G7/8 à ceux de l'UE ou de l'OCDE et de l'ONU. Ces trajectoires professionnelles valident leur statut d'experts, tout en faisant circuler leurs idées, leurs croyances et leurs pratiques. A cet égard, on peut admettre que «la constitution d'une communauté internationale d'experts qui partagent un même vocabulaire technique représente l'un des principaux acquis de la lutte anti-blanchiment ${ }^{38}$ ». C'est en ce sens qu'on peut appréhender la cohérence apparente du régime global anti-blanchiment tel qu'il a été conçu. On comprend ainsi mieux l'effet de circulation des normes en matière de criminalité financière, notamment entre le G8, le GAFI, et l'UE. Mais cet effet de mobilisation et de 
mobilité ne se fait pas sans clivages, et dire que ces acteurs se rencontrent de manière récurrente dans des instances diverses, facilitant ainsi une circulation des normes, ne suffit pas à comprendre la teneur de ce régime global anti-blanchiment. Car ces acteurs de la mobilisation sont loin de constituer un tout homogène; ils appartiennent à des bureaucraties variées, ont un savoir et des ressources particulières. C'est cet aspect qu'il faut désormais approfondir, afin de saisir l'apparent consensus que semble refléter ce régime global qui s'est imposé, peut-être au détriment d'autres alternatives.

Un régime global anti-blanchiment : ses éléments de justification et les outils mis en oeuvre

La multiplicité de ces lieux d'échanges et de rencontres a contribué à la mise en place de réseaux internationaux d'experts, qui ont permis une certaine harmonisation des pratiques anti-blanchiment au niveau international. L'actualisation de ces pratiques se trouvait justifiée, à l'origine, par un discours dominant qui s'est imposé au cours des années 1990 dans l'ensemble des institutions (aux niveaux régional, national et international) sur le blanchiment d'argent. La justification d'un régime global antiblanchiment s'est en effet opérée à travers un discours qui s'est largement uniformisé à l'échelle internationale, mais qui a évolué dans le temps. Comme le souligne Valsamis Mitsilegas ${ }^{39}$, la première tentative pour contrer le blanchiment d'argent a été entreprise dans le contexte de l'intensification de la "guerre contre la drogue » et sous l'influence des Etats-Unis dans les années 1980. Puis elle a été amplifiée par la volonté de préserver les systèmes financiers et banquiers de la pénétration de cet argent sale. Dans les années 1990, l'émergence dans les discours politiques de la question du crime organisé comme l'une des menaces les plus sérieuses pesant sur l'ordre international conduit à une approche plus globale de la lutte anti-blanchiment, qui se doit de tenir compte de cette forme multiple de criminalité. Enfin, les attentats du 11 septembre 2001 font $\mathrm{du}$ financement $\mathrm{du}$ terrorisme la nouvelle cible de la lutte contre le blanchiment d'argent ${ }^{40}$. Reconnu comme le «moteur» qui rend possible les actions criminelles de toutes sortes, celui-ci a été ainsi érigé en menace transnationale et le combat mené contre lui a été légitimé par le postulat que les organisations criminelles et les terroristes ne peuvent opérer sans l'accès à des ressources financières et qu'ils exploitent les faiblesses existantes des contrôles internationaux des systèmes financiers ${ }^{41}$. Même si la cible de la lutte anti-blanchiment a évolué, son objectif est sensiblement resté le même : couper les vivres aux organisations criminelles afin de les rendre moins opérationnelles et créer par là un effet de dissuasion. C'est à partir de ce consensus minimal entre acteurs policiers, judiciaires, financiers publics ou privés, que s'est progressivement construite la lutte anti-blanchiment au niveau international ${ }^{42}$. L'un des effets de ce consensus a été d'harmoniser l'extension de l'incrimination. L'incrimination du blanchiment à d'autres crimes que le trafic de stupéfiants, étendue aux crimes " graves » en général, est une tendance qui s'est vérifiée à la fois au sein de la Commission européenne (dans sa deuxième directive amendée de $2001{ }^{43}$ et sa troisième version de $2004{ }^{44}$ ) et au sein du Conseil de l'Europe (Convention de 1990 relative au blanchiment de capitaux, le dépistage, la saisie et la confiscation des produits du crime). Ensuite, l'adoption par le GAFI, juste après les attentats du 11 septembre, de neuf recommandations spéciales ${ }^{45}$ visant la lutte contre le financement du terrorisme a largement trouvé écho au sein de l'ONU avec la résolution 1373, et de la Commission européenne avec ses directives de 2001, et de 2004. Aujourd'hui, on trouve des outils reconnus comme nécessaires et efficaces dans la lutte contre le blanchiment de gains illégaux. Outre le champ d'application de l'infraction, qui s'est étendu et 
harmonisé au sein de ce régime global, deux éléments centraux sont identifiables : le volet réactif à l'infraction et le volet préventif. Le volet réactif fait intervenir des mesures de confiscation des biens blanchis et des produits des biens qui en découlent, le gel et la saisie de ces biens au cours des enquêtes. Le volet préventif s'étend au secteur privé, avec l'implication des institutions financières : règles d'identification des clients et de

conservation des documents, mais aussi une incitation à une « diligence accrue » de ces institutions financières, c'est-à-dire l'obligation d'agir de manière proactive à l'aide de déclarations de soupçons si des transactions semblent suspectes. Depuis le 11 septembre, ces dispositifs se sont étendus à d'autres professions, notamment libérales (comme les avocats). Bien que ces dispositifs aient auparavant rencontré des obstacles dans certaines législations (notamment en ce qui concerne l'obligation du secret professionnel) ou des réticences, ils ont été adoptés au niveau international. Parmi les neuf recommandations spéciales du GAFI, sont également impliqués les organismes à but non lucratif. Ce consensus cache en revanche des lignes de fractures sur la conception de l'action à mener.

Un consensus fragilisé

31 Ce réseau d'acteurs internationaux a, certes, contribué à rendre possible la constitution d'un régime global anti-blanchiment. Il faut néanmoins bien se garder de considérer ce réseau comme un front uni contre un ennemi commun. Si l'argument «moral» l'a emporté, justifiant des méthodes et des pratiques uniformisées, il faut bien admettre que cette lutte anti-blanchiment a été aussi le théâtre de clivages et de compétitions. A ce niveau de l'analyse, les appartenances institutionnelles des acteurs de la mobilisation (que ce soit au sein de l'UE, du G8, de l'OCDE ou encore de l'ONU) perdent de leur profondeur explicative. Ce sont davantage les positionnements bureaucratiques et nationaux, les questions de formation et de background professionnel de ces mêmes acteurs qui priment. Si l'élaboration d'un régime anti-blanchiment a été possible, c'est en partie grâce aux échanges constants entre différentes bureaucraties aux niveaux national, régional et international. Cette élaboration n'a néanmoins pas empêché la prévalence d'intérêts complexes et divers. D'un côté, les acteurs financiers publics issus des ministères des Finances ont eu, dès le départ, un rôle majeur dans la lutte antiblanchiment, au nom de l'intégrité,des systèmes financiers. D'un autre côté, la lutte contre l'argent sale justifiant la coopération de plusieurs administrations (notamment la police et la justice, au nom de la lutte contre la criminalité), les outils adoptés au niveau international tendent à refléter cette coopération désirée mais à minima. L'équilibre fragile entre ces groupes d'acteurs et leurs intérêts propres (les acteurs financiers et la préservation des marchés financiers / les acteurs judiciaires et policiers pour l'action préventive et répressive contre les criminels) a créé un régime de compromis. L'harmonisation des règles nationales de contrôle des transactions financières, par exemple, ne s'est pas faite sans heurts, et l'implication d'acteurs financiers privés dans la lutte contre l'argent sale ${ }^{46}$, l'équilibre délicat entre libertés de mouvement des capitaux et contrôle des flux d'argent ${ }^{47}$ ont été autant de compromissions entre acteurs économiques publics ou privés, d'une part, et acteurs financiers et répressifs d'autre part. Ces compromissions successives expliquent d'ailleurs certaines incohérences du régime actuel anti-blanchiment, notamment son silence sur la problématique des infractions fiscales ou les diverses formes de délinquance d'affaires ${ }^{48}$. De même en est-il de l'inclusion du financement du terrorisme dans la problématique anti-blanchiment, qui bouleverse encore davantage les rapports 
entre acteurs publics et acteurs privés, à qui on assigne un rôle de plus en plus proactif. Il ne s'agit plus seulement des obligations faites aux établissements bancaires ; celles-ci touchent désormais également les casinos, les fiducies et prestataires de services pour les sociétés, et les professions reconnues comme sensibles (avocats, notaires, comptables, agents immobiliers). Ces nouvelles obligations intentent parfois aux libertés, comme c'est le cas pour l'implication des avocats. Cet aspect fait d'ailleurs l'objet de nombreuses protestations

et certains Etats ont refusé de transposer cette règle dans leur législation ${ }^{49}$. L'incorporation de la lutte contre le financement du terrorisme dans la lutte antiblanchiment ne s'est d'ailleurs pas faite sans difficultés, les acteurs étant divisés (au-delà des clivages bureaucratiques, mais aussi nationaux) sur son bien-fondé. Car le financement du terrorisme n'est pas nécessairement "criminel» et les sources financières peuvent en être parfaitement légales ${ }^{50}$. La ligne de démarcation entre gain légal / illégal devient, par conséquent, de plus en plus mince ${ }^{51}$ et rend extrêmement délicate la lutte contre le blanchiment d'argent, qui doit s'attaquer non seulement aux gains illicites, mais aussi aux gains licites s'ils sont aux mains d'organisations criminelles ou terroristes ${ }^{52}$. Enfin, si la démarche ici adoptée avait pour objectif de dégager les institutions impliquées dans le processus d'élaboration des normes antiblanchiment, en s'appuyant notamment sur le G7/8 et le GAFI, il convient de rappeler le rôle croissant des Institutions financières internationales (IFI) dans ce domaine, notamment la Banque mondiale et le FMI. Si ce sont bien les ministres des Finances du G7 qui sont parvenus à impulser l'uniformisation d'une méthodologie anti-blanchiment à la fois au sein du GAFI, du FMI et de la Banque mondiale, les IFI semblent de plus en plus s'autonomiser par rapport à cette tutelle d'origine et élaborer leurs propres mécanismes dans la lutte contre l'argent sale ${ }^{53}$. La démarche proposée avait pour but d'étudier et de mieux comprendre les normes internationales inhérentes au régime global anti-blanchiment. En ce sens, nous avions abordé ces normes en les définissant d'emblée comme des attentes collectives quant à un comportement adéquat d'acteurs dotés d'une identité propre et, ce, dans une perspective constructiviste. Si les normes antiblanchiment ont, certes, créé une attente collective quant à leur internalisation au sein des administrations concernées, il faut cependant reconnaître, à l'aune de ce qui été démontré, que les convergences qui se sont opérées au niveau international se sont constituées au prix de compromis, dont le résultat a été un consensus

33 a minima. Si la circulation d'experts internationaux, gravitant autour d'enceintes multiples a permis l'élaboration de ce régime global - et, dans cette perspective, il a été démontré que l'UE était loin d'être isolée dans ce mouvement - des lignes de fracture demeurent. Ces réflexions ramènent à une conception des normes qui se voudrait un peu plus complexe que ne le suggère la définition ici

utilisée pour les caractériser. Ce n'est pas parce que le blanchiment d'argent est reconnu comme un «mal » et que les arguments moraux ont été largement utilisés afin de justifier l'édiction d'un régime global, que s'effacent pour autant les intérêts des acteurs qui l'ont élaboré. Ces entrepreneurs de normes, même s'ils se rencontrent, même s'ils sont parfois liés et souvent complices, ne parviennent pas toujours à dépasser leurs clivages, d'ordre professionnel, bureaucratique, ou encore national. L'identification des normes ainsi ne suffit pas à en déduire leur intériorisation par les acteurs concernés ${ }^{54}$. 


\section{NOTES}

1.. Je tiens particulièrement à remercier Gilles Favarel-Garrigues pour ses précieux conseils.

2.. La première définition du blanchiment d'argent à portée internationale a été donnée dès 1988, dans la convention des Nations unies contre le trafic de stupéfiants et de substances psychotropes (Vienne, 19 décembre 1988). Elle s'élaborait essentiellement autour des notions de conversion ou de transfert de biens acquis de manière illégale. Elle sera reprise par la Convention de Strasbourg de 1990. La définition qu'en donne le GAFI dès 1990 se veut plus englobante : elle traite non seulement de la transformation de l'argent sale, mais aussi de son recel et de sa dissimulation, ainsi que l'acquisition et détention de biens dont l'acquéreur connaît leur origine illégale.

3.. Selon la définition qu'en donne Krasner S., International Regimes, Ithaca, Cornell, University Press, 1983, p. 2.

4.. Voir par exemple Reuter P., Truman E., Chasing Dirty Money, The Fight against Money Laundering, Institute for International Economics, Washington, 2004 ; Godefroy T., Lascoumes P., Le Capitalisme clandestin. L'illusoire régulation des places offshore, Paris, La Découverte, 2004 ; Naylor R.T., Wages of Crime. Black Markets, Illegal Finance and the Underworld Economy, Ithaca et Londres, Cornell University Press, 2002.

5.. On appréhendera ici les normes comme « des attentes collectives quant à un comportement adéquat d'acteurs dotés d'une identité propre ", selon la définition qu'en donne Katzenstein P., dans l'introduction à son ouvrage The Culture of National Security : Norms and Identity in World Politics, New York, Columbia University Press, 1996, p. 5. (Traduction de l'auteur)

6.. On parlera du G7/8 en y intégrant la Russie, présente aux sommets depuis le début des années 1990, mais n'ayant acquis son statut officiel de membre qu'en 1998. Il sera précisé G7 quand la Russie n'en faisait pas encore partie.

7.. Sommet du G7 de Paris, Déclaration économique, disponible en ligne : http:// www.g8.utoronto.ca/francais/1989paris/economique.html. L'ensemble des déclarations et communiqués du G7/8 sont accessibles en ligne : www.g8.utoronto.ca 8.. Déclaration finale du sommet de Naples. Disponible en ligne : http:// www.g8.utoronto.ca/francais/1994naples/economique.html 9.. Communiqué final du sommet de Denver, 1997. Disponible en ligne : http:// www.g8.utoronto.ca/francais/1997denver/communique.html

10.. Déclaration finale du sommet de Birmingham. Disponible en ligne : http:// www.g8.utoronto.ca/francais/1998birmingham/drogues.html

11.. Statement by the leaders of the G8 over last week's terrorist attacks in New York and Washington, 19 septembre 2001.

12.. Convention relative aux infractions et à certains autres actes survenant à bord des aéronefs signée à Tokyo, le 14 septembre 1963 ; Convention pour la répression de la capture illicite d'aéronefs, signée à La Haye, le 16 décembre 1970 ; Convention pour la répression d'actes illicites dirigés contre la sécurité de l'aviation civile, signée à Montréal, le 23 septembre 1971 ; Protocole pour la répression des actes illicites de violence dans les aéroports servant à l'aviation civile internationale signé à Montréal, le 24 février 1988 ; Convention sur la prévention et la répression des infractions contre les personnes jouissant d'une protection internationale, $\mathrm{y}$ compris les agents 
diplomatiques, adoptée par l'Assemblée générale des Nations unies, le 14 décembre 1973 ; Convention internationale contre la prise d'otages adoptée par l'Assemblée générale des Nations unies, le 18 décembre 1979 ; Convention sur la protection physique des matières nucléaires, signée à New York et à Vienne, le 3 mars 1980 ; Convention pour la répression d'actes illicites contre la sécurité de la navigation maritime, conclue à Rome, le 10 mars 1988 ; Protocole à la Convention susmentionnée pour la répression d'actes illicites contre la sécurité des plates-formes fixes situées sur le plateau continental, conclu à Rome, le 10 mars 1988 ; Convention sur le marquage des explosifs plastiques aux fins de détection signée à Montréal, le 1er mars 1991; Convention internationale pour la répression des attentats terroristes à l'explosif, adoptée par l'Assemblée générale des Nations unies, le 15 décembre 1997 ; Convention internationale pour la répression du financement du terrorisme, adoptée par l'Assemblée générale des Nations unies, le 15 décembre 1997.

13.. La trouvaille de cette dénomination demeure floue; elle fait en tout cas référence aux porteurs des hauteurs de l'Himalaya, rendus célèbres à travers le monde après l'ascension de l'Everest en 1953. Le terme « sherpa » est désormais largement répandu dans la structure G8 et renvoie aux représentants personnels des chefs d'Etat et de gouvernement des pays membres du G8.

14.. Pour plus de détails sur le fonctionnement du G7/8, voir Scherrer A., « Le G8 face au crime organisé », G8 Governance, février 2005, n¹1, disponible en ligne : http:// www.g8.utoronto.ca/governance/scherrer_g8g.pdf

15.. La Russie est exclue des réunions ministérielles des Finances des Sept. Toutefois, elle y est associée selon les thèmes traités, y compris quand il s'agit de la lutte contre le blanchiment d'argent.

16.. Action Plan to Combat the Financing of Terrorism, Déclaration des ministres des Finances du G7, 19 décembre 2001.

17.. Ces neuf recommandations sont venues compléter les quarante recommandations initiales du GAFI. Elles sont consultables sur le site du GAFI : www.fatf-gafi.org

18.. Réunion des ministres de l'Intérieur et de la Justice, Moscou, 19-20 octobre 1999.

19.. Déclaration des ministres des Finances et des gouverneurs des banques centrales du G7, Tokyo, 22 janvier 2000, version non officielle.

20.. Notamment, les experts du groupe de Lyon promeuvent le partage et l'échange de bonnes pratiques en ce qui concerne les méthodes d'enquête et les mesures de détection et de surveillance des mouvements des capitaux transfrontaliers, ainsi que la détection des réseaux financiers liés à la criminalité transnationale organisée et au terrorisme, voir G8 Recommendations on Transnational Crime, 2002.

21.. Le groupe d'experts sur le terrorisme fut créé en 1982 au sommet du G7 à Versailles.

22.. G8 Recommendations on Counter-Terrorism, 2002.

23.. Le GAFI comprend aujourd'hui trente et un pays et territoires, et sept organisations régionales type GAFI. Voir www.fatf-gafi.org.

24.. Risse T., Sikkink K., « The socialization of international human rights norms into domestic practices ", in Risse T. (ed.), The Power of Human Rights, International Norms and Domestic Change, Introduction, Cambridge, Cambridge University Press, 1999, pp. 1-38, et surtout p. 11.

25.. La France, l'Italie, l'Allemagne, la Grande-Bretagne, les Etats-Unis, le Canada et le Japon. 
26.. Cette liste noire a été mise en place par le GAFI en 2000. Elle consiste à repérer et à divulguer la liste des « mauvais élèves » dans la lutte contre le blanchiment d'argent. Son impact se fonde sur le principe du « naming-shaming ». Pour plus de détails, voir sur ce point Godefroy T., Lascoumes P., Le Capitalisme clandestin. L'illusoire régulation des places offshore, op. cit., p. 166 et suivantes.

27. Cette intégration a certes été prépondérante à la socialisation de la Russie au sein des concertations des pays du G7. Néanmoins, il ne faut pas attribuer les efforts russes à la seule volonté de rejoindre le groupe des Sept. L'élaboration de mécanismes antiblanchiment a également répondu à des intérêts nationaux. Voir en ce sens les travaux de Gilles Favarel-Garrigues, " “Crime organisé transnational” et lutte antiblanchiment ", in Laroche J. (ed.), Mondialisation et gouvernance mondiale, Paris, PUF, 2003, pp. 161-173, surtout p. 172, ainsi que « Domestic Reformulation of the Moral Issues at Stake in the Drive Against Money Laundering : the Case of Russia », ISSJ, UNESCO, 2005.

28.. Mitsilegas V., " Countering the Chameleon Threat of Dirty Money. "Hard" and "Soft" Law in the Emergence of a Global Regime Against Money Laundering and Terrorist Finance ", in Edwards A. et Gill P. (ed.), Transnational Organised Crime. Perspectives on Global Security, Routledge, 2003, pp. 195-211, surtout p. 199.

29.. Le Japon a aussi, en ce sens, son rôle d'influence dans les enceintes régionales asiatiques.

30.. Depuis 1977, le président de la Commission européenne est invité à participer aux sommets. Le président du Conseil européen fut invité, quant à lui, pour la première fois en 1982. Depuis, l'Union européenne envoie une délégation à tous les sommets et aux réunions ministérielles et participe au processus préparatoire.

31.. Appelée désormais « Justice, Liberté et Sécurité » (JLS).

32. Engueleguele S., « Les communautés épistémiques pénales et la production législative en matière criminelle ", Droit et Société, n40, 1998, pp. 563-581, p. 565. 33.. Haas P., « Introduction : Epistemic Communities and International Policy Coordination ", International Organization, vol.49, n¹, 1992, pp. 1-35.

34.. C'est ce qui ressort largement d'une série d'entretiens effectués au sein de certains départements des Affaires étrangères, notamment en France, au Canada et en GrandeBretagne.

35.. Nous rejoignons ici les travaux d'Yves Dezalay sur les propriétés sociales et culturelles de ces agents qui font profession de l'international. Voir Dezalay Y. et Garth B., La Mondialisation de la guerre des palais : la restructuration du pouvoir par l'Etat en Amérique Latine, entre notables du droit et « Chicago Boys », Paris, Le Seuil, 2002.

36. Comme c'est le cas en France avec le Bureau de la lutte contre la criminalité organisée, le terrorisme et le blanchiment.

37. L'Unité de coordination de la lutte anti-terroriste (UCLAT) pour le ministère de l'Intérieur

en France qui a intégré depuis peu l'UCRAM (l'Unité de coordination et de recherches antimafia), le Bureau de la coopération multilatérale de sécurité et de défense au sein de la gendarmerie nationale française, ou encore le tout nouveau SOCA (Serious Organised Crime Agency) britannique.

38. Favarel-Garrigues G., " “Crime organisé transnational” et lutte anti-blanchiment ", op. cit., pp. 161-173, p. 167. 
39.. Mitsilegas V., " Countering the Chameleon Threat of Dirty Money. "Hard" and "Soft" Law in the Emergence of a Global Regime Against Money Laundering and Terrorist Finance ", op. cit., pp. 195-211.

40.. Ibid., pp. 195-211.

41.. Ce discours qui s'est imposé est loin de faire l'unanimité. De nombreux écrits académiques en pointent les incohérences et les limites. Voir notamment FavarelGarrigues G., " "Crime organisé transnational” et lutte anti-blanchiment », op. cit., pp. 161-173 ; Lascoumes P., Godefroy T., op. cit. ; Naylor T., Beare M., « Major Issues Relating to Organized Crime », 1999, Law commission of Canada.

42.. Favarel-Garrigues G., " "Crime organisé transnational" et lutte anti-blanchiment ", op. cit., pp. 161-173, p. 171.

43.. 2001/97/CE, Directive du Parlement européen et du Conseil du 4 décembre 2001 modifiant la directive 91/308/CEE de Conseil relative à la prévention de l'utilisation du système financier aux fins de blanchiment de capitaux (JO L344, 29 décembre 2001, 76).

44.. Directive du Parlement européen et du Conseil relative à la prévention de l'utilisation du système financier aux fins de blanchiment de capitaux, y compris du financement du terrorisme, adoptée par le Parlement européen en 2005.

45.. Ces neuf recommandations prévoient, entre autres, l'incrimination du financement du terrorisme et du blanchiment de capitaux commis dans le cadre des activités terroristes, le gel et la confiscation des biens des terroristes, l'extension des déclarations des transactions suspectes liées au terrorisme.

46.. La lutte internationale anti-blanchiment a profondément modifié les rapports entre acteurs publics et privés. L'obligation de déclaration de soupçon des transactions suspectes par les établissements bancaires, le rôle proactif qui leur est assigné notamment la règle de « Know Your Customer » - créent de nouvelles contraintes. Voir sur ce point Mitsilegas V., op. cit., pp. 195-211, p. 196-200.

47.. On pointe ici le débat entre dérégulation des marchés financiers et re-régulations que ces contrôles étatiques impliquent sur le flux des capitaux au nom de la lutte contre l'argent sale. S'il y a eu un consensus entre acteurs privés et publics sur la nécessité de contrôler les flux illégaux émanant de criminels, il ne s'est pas étendu au thème de l'évasion fiscale. Voir sur ce point Helleiner E., « State Power and the Regulation of Illicit Activity in Global Finance », in Friman H., Andreas P. (ed), Illicit Global Economy and State Power, Lanham, Md. Rowman and LittleField, 1999, pp. 53-91. 48. La non-inclusion de cette délinquance d'affaires a été l'objet de nombreux écrits. Ce silence sur des acteurs privés plus « legitimes » que les trafiquants de drogues ou les terroristes est une problématique ancienne. Voir sur ce point Godefroy T., Lascoumes P., op. cit., notamment sur les affaires « Mediaset » (p. 74), « Executive Life » (p. 76) et «Enron» (p. 78) ; voir aussi dans une perspective historique Woodiwiss M., "Transnational Organized Crime : The Strange Career of an American Concept », in Beare M. (ed.), Critical Reflections on,Transnational Organized Crime, Money Laundering and Corruption, Toronto, University of Toronto Press, 2003, pp. 3-34.

49.. Mitsilegas V., op. cit., pp. 202-203.

50.. Ibid., pp.195-211, p. 207.

51.. Ibid., p. 208.

52. Van Duyne Petrus C., "Greasing the Organisation of crime-markets in Europe ", in Von Lample K., Newell James L. (ed.) Criminal Finances and Organised Crime in Europe, WLP, 2003, pp. 1-17. 
53.. Favarel-Garrigues G., " "Crime organisé transnational” et lutte anti-blanchiment », op. cit., pp. 161-173, p. 166-167 et « Le renouvellement des listes noires contre l'argent sale ", à paraître dans la revue Criminologie en 2006, où l'auteur montre la montée en puissance des IFI dans le domaine de la lutte anti-blanchiment et le confinement progressif du GAFI à un rôle de « standards setting » ainsi que l'abandon de ses fameuses « listes noires ».

54.. Nous faisons ici écho à l'article de David Ambrosetti sur les normes humanitaires. Voir « L'humanitaire comme norme du discours au Conseil de sécurité : une pratique légitimatrice socialement sanctionnée ", Cultures \& Conflits, n60, 2005, pp. 39-62.

\section{RÉSUMÉS}

Cet article se propose d'analyser le rôle du G7/8 dans le régime global anti-blanchiment d'argent. Il s'attache à dégager les mécanismes de cette instance mal connue qui ont contribué à la mise en place de normes au niveau international contre le blanchiment d'argent, et plus récemment contre le financement du terrorisme. En ce sens, il sera montré comment des normes particulières ont circulé sur la scène internationale, entre diverses institutions, et notamment entre l'Union européenne et le G7/8. Cette circulation a été largement facilitée par des acteurs engagés dans cette mobilisation anti-blanchiment, et plus particulièrement ces " experts » des administrations publiques des pays membres de ces institutions. Une approche sociologique permettra de mettre en valeur les trajectoires professionnelles de ces experts, trajectoires qui expliquent ces lignes de convergence, mais aussi de clivage qui ont façonné le régime global antiblanchiment tel qu'il apparaît aujourd'hui.

This article aims at describing the role of the G7/8 in the anti-money laundering global regime. It will provide an insight of the G7/8 mechanisms that are often not clearly understood and have contributed to the elaboration of norms against money laundering and, more recently, terrorist financing. The goal is then to give an understanding of those specific norms, which have circulated among several international institutions, in particular in the European Union and the G7/8. The diffusion of these norms has been facilitated by actors engaged in the global mobilisation against money-laundering, specifically by those "experts" of Member States' public administrations. A sociological approach will be used to look at these experts' professional background, highlighting convergences, but also divergences that have framed the existing antimoney laundering global regime.

\section{INDEX}

Mots-clés : coopération internationale, droit international, lutte contre la criminalité organisée, normes internationales, organisations internationales

Thèmes : G7/ G8 


\section{AUTEUR}

\section{AMANDINE SCHERRER}

Amandine Scherrer est doctorante en Relations internationales au CERI. Ses recherches portent sur la production normative du G8 face à la criminalité transnationale organisée. 